\title{
Improvement of Nemhauser-Trotter Theorem and its applications in parametrized complexity
}

\author{
Miroslav Chlebík ${ }^{1}$ and Janka Chlebíková2 * \\ 1 Max Planck Institute for Mathematics in the Sciences \\ Inselstraße 22-26, D-04103 Leipzig, Germany \\ chlebik@mis.mpg.de \\ 2 Institut für Informatik und Praktische Mathematik \\ CAU, Olshausenstraße 40, D-24098 Kiel, Germany \\ jch@informatik.uni-kiel.de
}

\begin{abstract}
We improve on the classical Nemhauser-Trotter Theorem, which is a key tool for the Minimum (Weighted) Vertex Cover problem in the design of both, approximation algorithms and exact fixedparameter algorithms. Namely, we provide in polynomial time for a graph $G$ with vertex weights $w: V \rightarrow\langle 0, \infty)$ a partition of $V$ into three subsets $V_{0}, V_{1}, V_{\frac{1}{2}}$, with no edges between $V_{0}$ and $V_{\frac{1}{2}}$ or within $V_{0}$, such that the size of a minimum vertex cover for the graph induced by $V_{\frac{1}{2}}$ is at least $\frac{1}{2} w\left(V_{\frac{1}{2}}\right)$, and every minimum vertex cover $C$ for $(G, w)$ satisfies

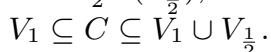

We also demonstrate one of possible applications of this strengthening of NT-Theorem for fixed parameter tractable problems related to MiN-VC: for an integer parameter $k$ to find all minimum vertex covers of size at most $k$, or to find a minimum vertex cover of size at most $k$ under some additional constraints.
\end{abstract}

\section{Introduction}

The (weighted) Vertex Cover problem (shortly, Min-w-VC) is one of the fundamental NP-hard problems in the combinatorial optimization. In spite of a great deal of efforts, the tight bound on its approximability by a polynomial time algorithm is left open. Recall that the problem has a simple 2-approximation algorithm and currently the best lower bound on polynomial time approximability is $10 \sqrt{5}-21 \approx 1.36067$, due to Dinur and Safra [7]. The parametrized version of the VERTEX COVER problem is a well known fixed parameter tractable (FPT) problem and has received considerable interest: for a given graph and a positive integer $k$, the problem is to find a vertex cover of weight at most $k$ or to report that no such vertex cover exists.

A key tool for the approximation algorithms and for the parametrized version of MiN- $w$-VC is the Nemhauser-Trotter Theorem (NT-Theorem). The NTTheorem efficiently reduces the MIN- $w$-VC problem to instances $(G, w)$, in which

* Supported by the EU-Project ARACNE, Approximation and Randomized Algorithms in Communication Networks, HPRN-CT-1999-00112. 
the size of a minimum vertex cover is at least $\frac{1}{2} w(V)$. This result is useful as an approximation preserving preprocessing step, that reduces the problem to find one optimal vertex cover to more restricted instances. In the parametrized MiN-VC any kernelization technique reduces in conjunction or independently both, the size of the input graph and the parameter size. As observed in [4], NT-Theorem allows to find efficiently a linear size problem kernel for Min-VC.

But the original NT-Theorem and also other known kernelization techniques (see [1], [15]) are less efficient if for a graph $(G, w)$ and an integer parameter $k$ the goal is to find all minimum vertex covers of weight at most $k$, or to find a minimum vertex cover of weight at most $k$ under some additional constraints. In this contribution we overcome this difficulty and prove the theorem much stronger than the classical NT-Theorem. We also show how this result can be applied to obtain new algorithmic and complexity results for fixed-parameter tractable problems related to the MiN-w-VC (unweighted, for simplicity).

Preliminaries. Let $G=(V, E)$ be a graph with vertex weights $w: V \rightarrow\langle 0, \infty)$. For a set of vertices $U \subseteq V$, let $\Gamma(U):=\{v \in V: \exists u \in U$ such that $\{u, v\} \in E\}$ stand for the set of its neighbors, and $G[U]$ denote the subgraph of $G$ induced by $U$. The weight of a vertex subset $U \subseteq V$ is defined by $w(U):=\sum_{u \in U} w(u)$.

Minimum Weighted Vertex Cover (Min- $w$-VC)

Instance: A simple graph $G=(V, E)$ with vertex weights $w: V \rightarrow\langle 0, \infty)$.

Feasible solution: A vertex cover $C$ for $G$, i.e., a subset $C \subseteq V$ such that for each $e \in E, e \cap C \neq \emptyset$.

Objective function: The weight $w(C):=\sum_{u \in C} w(u)$ of the vertex cover $C$.

The unweighted version the Minimum Vertex Cover problem (shortly, Min-VC) is the special case of Min- $w$-VC with uniform weights $w(u)=1$ for each $u \in V$. Let $\operatorname{VC}(G, w)$ be the set of all minimum vertex covers for $(G, w)$ and $v c(G, w)$ stand for the weight of the minimum vertex cover for $(G, w)$. In unweighted case we use shortly $V C(G)$ and $v c(G)$.

MiN- $w$-VC problem can be expressed as an Integer Program (IP) as follows: the goal is to minimize the function $w(x):=\sum_{u \in V} w(u) \cdot x(u)$, where $x(u) \in$ $\{0,1\}$ for each $u \in V$, and a feasible solution $x: V \rightarrow\{0,1\}$ has to satisfy edge constraints $x(u)+x(v) \geq 1$ for each edge $\{u, v\} \in E$. There is one-to-one correspondence between the set of vertex covers for $G$ and the set of functions $x: V \rightarrow\{0,1\}$ satisfying all edge constraints; each such $x$ is an indicator function of some vertex cover for $G$.

The Linear Programming (LP) relaxation of the IP-formulation allows $x(u) \in$ $\langle 0,1\rangle$ (or even $x(u) \geq 0$ ). It is well known ([12], [14]) that there always exists an optimal solution of the LP-relaxation with the variables $x(u) \in\left\{0, \frac{1}{2}, 1\right\}$. The Half-Integral (HI) relaxation has exactly the same formulation as the IPformulation, but it allows variables $x(u)$ from the set $\left\{0, \frac{1}{2}, 1\right\}$ for each $u \in V$. Hence a feasible solution is a half-integral vertex cover for $G$, i.e., a function $x: V \rightarrow\left\{0, \frac{1}{2}, 1\right\}$ satisfying edge constraints $x(u)+x(v) \geq 1$ for each edge $\{u, v\} \in E$. Let $V C^{*}(G, w)$ be the set of all minimum half-integral vertex covers $x: V \rightarrow\left\{0, \frac{1}{2}, 1\right\}$, and $v c^{*}(G, w)$ stand for the weight of a minimum half-integral 
vertex cover for $(G, w)$. For a minimum half-integral vertex cover $x$ for $(G, w)$, we denote $V_{i}^{x}:=\{u \in V: x(u)=i\}$ for each $i \in\left\{0, \frac{1}{2}, 1\right\}$.

Clearly, $v c^{*}(G, w) \leq v c(G, w)$, as for any vertex cover $C$ its indicator function $x^{C}$ is a feasible solution for the HI-relaxed problem with $w\left(x^{C}\right)=w(C)$. Further, $v c^{*}(G, w) \leq \frac{1}{2} w(V)$, as the function $x \equiv \frac{1}{2}$ on $V$ is always feasible solution for the HI-relaxation. The (weighted) graphs $G=(V, E)$ for which the equality $v c^{*}(G, w)=\frac{1}{2} w(V)$ holds play a special role in the Min- $w$-VC problem. All the difficulty solving the problem exactly, or approximating it, reduces to such graphs.

Overview. The main goal of Section 2 is to provide the following strengthened version of Nemhauser-Trotter Theorem, its full version with additional properties is contained in Theorem 2 .

Optimal version of Nemhauser-Trotter Theorem. There exists a polynomial time algorithm that partitions vertex set $V$ of any graph $(G, w)$ with vertex weights $w: V \rightarrow\langle 0, \infty)$ into three subsets $V_{0}, V_{1}, V_{\frac{1}{2}}$ with no edges between $V_{0}$ and $V_{\frac{1}{2}}$ or within $V_{0}$ such that (i) $v c\left(G\left[V_{\frac{1}{2}}\right], w\right) \geq \frac{1}{2} w\left(V_{\frac{1}{2}}\right)$; and (ii) every minimum vertex cover $C$ for $(G, w)$ satisfies $V_{1} \subseteq C \subseteq V_{1} \cup V_{\frac{1}{2}}$ and $C \cap V_{\frac{1}{2}}$ is a minimum vertex cover for $\left(G\left[V_{\frac{1}{2}}\right], w\right)$.

The main difference is that the condition (ii) will be satisfied for every minimum vertex cover for $(G, w)$, not merely for some of them. Such result was known only in unweighted bipartite case, when it follows from matching theory (Gallai-Edmonds structure theorem).

The key point of this improvement is that one minimum half-integral vertex cover, called pivot, induces a decomposition $V=V_{0} \cup V_{1} \cup V_{\frac{1}{2}}$ that has the desired additional quality. In this case the kernel $V_{\frac{1}{2}}$, to which the problem is reduced, is the largest among all $V_{\frac{1}{2}}^{y}, y \in V C^{*}(G, w)$, in fact $V_{\frac{1}{2}}=\cup_{y \in V C^{*}(G, w)} V_{\frac{1}{2}}^{y}$. It is also crucial for applications that the pivot can be found efficiently.

In Section 3 we provide useful decomposition of a weighted graph $(G, w)$ that reflects the structure of the set of all optimal solutions for the HI-relaxed problem. This decomposition into "irreducible parts" carries information how all minimum vertex covers for $(G, w)$ are structured outside the minimal relevant kernel $K:=\cap_{y \in V C^{*}(G, w)} V_{\frac{1}{2}}^{y}$. As a simple byproduct we obtain a polynomial time algorithm that decides whether a minimum vertex cover has the same weight as the optimum of the HI-relaxed (equivalently, LP-relaxed) problem, and if yes, finds one minimum vertex cover for $(G, w)$.

In Section 4 we demonstrate one possible application, namely how our improvement of NT-Theorem can be used for fixed-parameter tractable problems related to the Min-VC problem (unweighted, for simplicity). The strengthening of NT-Theorem can be used as efficient reduction to linear size problem kernel even in situations in which NT-Theorem is less efficient, e.g., if the task is to find all minimum vertex covers for $G$ if $v c(G) \leq k$, or to report that $v c(G)>k$. Similarly, assuming $v c(G) \leq k$, to find a minimum vertex cover for $G$ under some additional constraints. 


\section{The Optimal Version of Nemhauser-Trotter Theorem}

In the following series of lemmas we study the basic properties of minimum halfintegral vertex covers. Recall that for a minimum half-integral vertex cover $x$ for $(G, w) V_{i}^{x}:=\{u \in V: x(u)=i\}$ for each $i \in\left\{0, \frac{1}{2}, 1\right\}$.

A minimum half-integral vertex cover $x$ with the property that $V_{\frac{1}{2}}^{x}$ is not a proper subset of $V_{\frac{1}{2}}^{y}$ for any $y$ from $V C^{*}(G, w)$, is called a pivot. It plays a special role in what follows.

Lemma 1. Given a graph $G=(V, E)$ with vertex weights $w: V \rightarrow\langle 0, \infty)$, and a partition $V=V_{0}^{x} \cup V_{1}^{x} \cup V_{\frac{1}{2}}^{x}$ according to a fixed minimum half-integral vertex cover $x$ for $(G, w)$. Then

(i) $V_{0}^{x}$ is an independent set, $\Gamma\left(V_{0}^{x}\right) \subseteq V_{1}^{x}$, and the vertices of $V_{1}^{x} \backslash \Gamma\left(V_{0}^{x}\right)$ have weight 0 .

(ii) If $x$ is a pivot then $V_{0}^{x}$ does not contain vertices of weight 0 , and $V_{1}^{x}=$ $\Gamma\left(V_{0}^{x}\right)$.

(iii) $v c^{*}\left(G\left[V_{\frac{1}{2}}^{x}\right], w\right)=\frac{1}{2} w\left(V_{\frac{1}{2}}^{x}\right)$

(iv) For each $U \subseteq V_{1}^{x}, w\left(\Gamma(U) \cap V_{0}^{x}\right) \geq w(U)$. If $x$ is a pivot, then $\emptyset \neq U \subseteq V_{1}^{x}$ implies $w\left(\Gamma(U) \cap V_{0}^{x}\right)>w(U)$.

Lemma 2. Given a graph $G=(V, E)$ with vertex weights $w: V \rightarrow\langle 0, \infty)$, and a partition $V=V_{0}^{x} \cup V_{1}^{x} \cup V_{\frac{1}{2}}^{x}$ according to a fixed minimum half-integral vertex cover $x$ for $(G, w)$. Then

(i) $V_{1}^{x}$ is a minimum vertex cover for $\left(G\left[V_{0}^{x} \cup V_{1}^{x}\right], w\right)$, hence $v c\left(G\left[V_{0}^{x} \cup V_{1}^{x}\right], w\right)=$ $w\left(V_{1}^{x}\right)$. If $x$ is a pivot, then $V_{1}^{x}$ is the unique minimum vertex cover for $\left(G\left[V_{0}^{x} \cup V_{1}^{x}\right], w\right)$.

(ii) $\left.x\right|_{V_{0}^{x} \cup V_{1}^{x}}$ is a minimum half-integral vertex cover for $\left(G\left[V_{0}^{x} \cup V_{1}^{x}\right], w\right)$, hence $v c^{*}\left(G\left[V_{0}^{x} \cup V_{1}^{x}\right], w\right)=w\left(V_{1}^{x}\right)$. If $x$ is a pivot, then $\left.x\right|_{V_{0}^{x} \cup V_{1}^{x}}$ is the unique minimum half-integral vertex cover for $\left(G\left[V_{0}^{x} \cup V_{1}^{x}\right], w\right)$.

Lemma 3. Given a graph $G=(V, E)$ with vertex weights $w: V \rightarrow\langle 0, \infty)$, and a partition $V=V_{0}^{x} \cup V_{1}^{x} \cup V_{\frac{1}{2}}^{x}$ according to a fixed minimum half-integral vertex cover $x$ for $(G, w)$. Then the following holds:

(i) Every (minimum) vertex cover for $\left(G\left[V_{\frac{1}{2}}^{x}\right], w\right)$ together with $V_{1}^{x}$ forms a (minimum) vertex cover for $(G, w)$. Every (minimum) half-integral vertex cover for $\left(G\left[V_{\frac{1}{2}}^{x}\right], w\right)$ extended by 1 on $V_{1}^{x}$ and by 0 on $V_{0}^{x}$ forms a (minimum) half-integral vertex cover for $(G, w)$.

(ii) For every minimum vertex cover $C$ for $(G, w), C \cap V_{\frac{1}{2}}^{x}$ and $C \cap\left(V_{0}^{x} \cup V_{1}^{x}\right)$ are minimum vertex covers for $\left(G\left[V_{\frac{1}{2}}^{x}\right], w\right)$ and $\left(G\left[V_{0}^{x} \cup V_{1}^{x}\right], w\right)$, respectively. For every minimum half-integral vertex cover y for $(G, w),\left.y\right|_{V_{\frac{1}{2}}^{x}}$ and $\left.y\right|_{\left(V_{0}^{x} \cup V_{1}^{x}\right)}$ are minimum half-integral vertex covers for $\left(G\left[V_{\frac{1}{2}}^{x}\right], w\right)$ and ${ }^{2}\left(G\left[V_{0}^{x} \cup V_{1}^{x}\right], w\right)$, respectively. 
(iii) If $x$ is a pivot, then for every minimum vertex cover $C$ for $(G, w)$ it holds $V_{1}^{x} \subseteq C \subseteq V_{1}^{x} \cup V_{\frac{1}{2}}^{x}$. Analogously for every minimum half-integral vertex cover $y$ for $(G, w)$ it holds $\left.y\right|_{V_{0}^{x}} \equiv 0$ and $\left.y\right|_{V_{1}^{x}} \equiv 1$.

In a bipartite graph a minimum vertex cover may be identified from the solution of the corresponding Minimum CUT problem. It can be found by efficient algorithms for the MAXIMUM FLOW problem on bipartite graphs (see Lawler [11]). For instance, the problem is solvable in time $O\left(|E||V| \log \frac{|V|^{2}}{|E|}\right)$ using Goldberg and Tarjan's algorithm [9]. When the problem is unweighted, Dinic's algorithm for the Maximum FLow problem runs in $O(|E| \sqrt{|V|})$ time. Another approach in unweighted case is based on the bipartite graph matching theory. A maximum matching of a bipartite graph can be constructed in time $O(|E| \sqrt{|V|}$ ) by the algorithm of Hopcroft and Karp ([10]) (or even for general graphs by the algorithm of Micali and Varizani), and a minimum vertex cover for a bipartite graph can be constructed from a maximum matching in linear time.

In what follows we define for a graph $(G, w)$ its weighted bipartite version $\left(G^{b}, w^{b}\right)$ and observe that the optimal solutions for the HI-relaxation of the MiN- $w$-VC problem for $(G, w)$ are generated by minimum weight covers for the corresponding bipartite graph $\left(G^{b}, w^{b}\right)$.

Definition 1. For a graph $G=(V, E)$ with vertex weights $w: V \rightarrow\langle 0, \infty)$ we define weighted bipartite version $\left(G^{b}, w^{b}\right)$, with $G^{b}=\left(V^{b}, E^{b}\right)$, as follows: there are two copies $u^{L}$ and $u^{R}$ of each vertex $u \in V$ of the same weight $w^{b}\left(u^{L}\right)=$ $w^{b}\left(u^{R}\right)=w(u)$ in $\left(G^{b}, w^{b}\right), V^{L}:=\left\{u^{L}: u \in V\right\}, V^{R}:=\left\{u^{R}: u \in V\right\}$, and $V^{b}:=V^{L} \cup V^{R}$. Each edge $\{u, v\} \in E$ of $G$ creates two edges in $G^{b}$, namely $\left\{u^{L}, v^{R}\right\}$ and $\left\{v^{L}, u^{R}\right\}$. Hence $E^{b}:=\left\{\left\{u^{L}, v^{R}\right\},\left\{v^{L}, u^{R}\right\}:\{u, v\} \in E\right\}$. For $U \subseteq$ $V$ we use also $U^{L}, U^{R}$, and $U^{b}:=U^{L} \cup U^{R}$ for the corresponding sets of vertices.

For any set $C \subseteq V^{\mathrm{L}} \cup V^{\mathrm{R}}$ we associate a map $x_{C}: V \rightarrow\left\{0, \frac{1}{2}, 1\right\}$ in the following way: $x_{C}(u)=\frac{1}{2}\left|C \cap\left\{u^{\mathrm{L}}, u^{\mathrm{R}}\right\}\right|$ for any $u \in V$. Clearly $w\left(x_{C}\right)=\frac{1}{2} w^{b}(C)$ for any $C \subseteq V^{\mathrm{L}} \cup V^{\mathrm{R}}$.

Lemma 4. (i) If $C$ is a vertex cover for $G^{b}$ then $x_{C}$ is a half-integral vertex cover for $G$ of weight $\frac{1}{2} w^{b}(C)$. In particular, $v c^{*}(G, w) \leq \frac{1}{2} v c\left(G^{b}, w^{b}\right)$.

(ii) If $x: V \rightarrow\left\{0, \frac{1}{2}, 1\right\}$ is a half-integral vertex cover for $G$ then there is a vertex cover $C$ for $G^{b}$ such that $x_{C}=x$. Hence $\frac{1}{2} v c\left(G^{b}, w^{b}\right) \leq v c^{*}(G, w)$.

(iii) $v c^{*}(G, w)=\frac{1}{2} v c\left(G^{b}, w^{b}\right)$

(iv) The mapping $C \mapsto x_{C}$ maps $\operatorname{VC}\left(G^{b}, w^{b}\right)$ onto $V C^{*}(G, w)$.

For a weighted graph $(G, w)$ the existence of a pivot is clear from its definition. By Lemma 1(ii), a pivot $x$ is determined by its $V_{0}^{x}$ part, as then $V_{1}^{x}=\Gamma\left(V_{0}^{x}\right)$ and $V_{\frac{1}{2}}^{x}=V \backslash\left(V_{0}^{x} \cup V_{1}^{x}\right)$. But Lemma 3(iii) implies that for every $y \in V C^{*}(G, w)$ $V_{0}^{x} \subseteq V_{0}^{y}, V_{1}^{x} \subseteq V_{1}^{y}$, and $V_{\frac{1}{2}}^{x} \supseteq V_{\frac{1}{2}}^{y}$, hence $V_{0}^{x} \subseteq \cap_{y \in V C^{*}(G, w)} V_{0}^{y}\left(\subseteq V_{0}^{x}\right), V_{1}^{x} \subseteq$ $\cap_{y \in V C^{*}(G, w)} V_{1}^{y}\left(\subseteq V_{1}^{x}\right)$, and $V_{\frac{1}{2}}^{x} \supseteq \cup_{y \in V C^{*}(G, w)} V_{\frac{1}{2}}^{y}\left(\supseteq V_{\frac{1}{2}}^{x}\right)$. Therefore the pivot $x$ is unique and it defines the partition with $V_{0}^{x}=V_{0}^{*}(G, w):=\cap_{y \in V C^{*}(G, w)} V_{0}^{y}$, 
$V_{1}^{x}=V_{1}^{*}(G, w):=\cap_{y \in V C^{*}(G, w)} V_{1}^{y}$, and $V_{\frac{1}{2}}^{x}=V_{\frac{1}{2}}^{*}(G, w):=V \backslash\left(V_{0}^{*}(G, w) \cup\right.$ $\left.V_{1}^{*}(G, w)\right)$.

Denote the analogous parts of $V$ corresponding to the set $V C(G, w)$ of minimum vertex covers as $V_{i}(G, w), i \in\left\{0,1, \frac{1}{2}\right\}$, where $V_{0}(G, w)$ is the set of vertices avoided by each minimum vertex cover for $(G, w), V_{1}(G, w)$ is the set of vertices contained in each minimum vertex cover for $(G, w)$, and $V_{\frac{1}{2}}(G, w)$ is the rest.

Remark 1. For a fixed weighted graph $(G, w)$ let $\Phi: V^{b} \rightarrow V^{b}$ denote the automorphism of $\left(G^{b}, w^{b}\right)$ defined by $\Phi\left(u^{\mathrm{L}}\right)=u^{\mathrm{R}}, \Phi\left(u^{\mathrm{R}}\right)=u^{\mathrm{L}}$ for each $u \in V$. For a fixed $u \in V$ we obtain $u^{\mathrm{L}} \in V_{0}\left(G^{b}, w^{b}\right)$ iff $u^{\mathrm{R}} \in V_{0}\left(G^{b}, w^{b}\right)$ iff (using Lemma 4) $u \in V_{0}^{*}(G, w) ; u^{\mathrm{L}} \in V_{1}\left(G^{b}, w^{b}\right)$ iff $u^{R} \in V_{1}\left(G^{b}, w^{b}\right)$ iff $u \in V_{1}^{*}(G, w)$. In other words, for each $i \in\left\{0,1, \frac{1}{2}\right\}, V_{i}\left(G^{b}, w^{b}\right)$ consists of pairs corresponding to vertices of $V_{i}^{*}(G, w)$.

Hence the set $V_{0}^{x}=V_{0}^{*}(G, w)$ for the pivot $x$ can be identified from $V_{0}\left(G^{b}, w^{b}\right)$, which can be computed efficiently due to the following lemma. Recall that for an unweighted bipartite graph it follows from the bipartite version of the classical Gallai-Edmonds Structure Theorem that the set $V_{0}$ coincides with the set of vertices avoided by at least one maximum matching.

Lemma 5. Let $G=(V, E)$ be a bipartite graph with vertex weights $w: V \rightarrow$ $\langle 0, \infty)$. The problem to find the set $V_{0}$, the set of all vertices in $G$ that are avoided by each minimum vertex cover for $(G, w)$, is solvable in polynomial time; in unweighted case in time $O(|E| \sqrt{|V|})$.

Clearly, with the set $V_{0}^{x}$ known, the set $V_{1}^{x}=\Gamma\left(V_{0}^{x}\right)$ and $V_{\frac{1}{2}}^{x}$ can be easily constructed for the pivot $x$. Therefore, we obtain the following theorem

Theorem 1. Let a graph $G=(V, E)$ with vertex weights $w: V \rightarrow\langle 0, \infty)$ be given. Among minimum half-integral vertex covers for $(G, w)$ there is exactly one pivot $x$. The corresponding partition $V=V_{0}^{x} \cup V_{1}^{x} \cup V_{\frac{1}{2}}^{x}$ according to $x$ has the following properties: $V_{0}^{x}=\cap_{y \in V C^{*}(G, w)} V_{0}^{y}, V_{1}^{x}=\cap_{y \in V C^{*}(G, w)} V_{1}^{y}=\Gamma\left(V_{0}^{x}\right)$, and $V_{\frac{1}{2}}^{x}=\cup_{y \in V C^{*}(G, w)} V_{\frac{1}{2}}^{y}$. Moreover, there is a polynomial time algorithm that finds the pivot $x$. In the unweighted case, its running time is $O(|E| \sqrt{|V|})$.

Remark 2. If $G=(V, E)$ is a bipartite graph with bipartition $V=A \cup B$ and with vertex weights $w: V \rightarrow\langle 0, \infty)$, then $\left(G^{b}, w^{b}\right)$ consists of two disjoint copies of $(G, w)$, namely $\left(G^{b}\left[A^{\mathrm{L}} \cup B^{\mathrm{R}}\right], w^{b}\right)$ and $\left(G^{b}\left[A^{\mathrm{R}} \cup B^{\mathrm{L}}\right], w^{b}\right)$. Therefore $v c\left(G^{b}, w^{b}\right)=$ $2 v c(G, w)$, and $v c^{*}(G, w)=v c(G, w)$ by Lemma 4(iii). Moreover, $u \in V_{0}(G, w)$ iff $u^{\mathrm{L}}, u^{\mathrm{R}} \in V_{0}\left(G^{b}, w^{b}\right)$ iff $u \in V_{0}^{*}(G, w)$, hence $V_{0}(G, w)=V_{0}^{*}(G, w)$. In the same way we get $V_{1}(G, w)=V_{1}^{*}(G, w)$.

We can summarize our previous results as follows:

Theorem 2. There exists a polynomial time algorithm that partitions the vertex set $V$ of a given graph $(G, w)$ with vertex weights $w: V \rightarrow\langle 0, \infty)$ into three subsets $V_{0}, V_{1}, V_{\frac{1}{2}}$ with no edges between $V_{0}$ and $V_{\frac{1}{2}}$ or within $V_{0}$, such that 
(i) $v c\left(G\left[V_{\frac{1}{2}}\right], w\right) \geq v c^{*}\left(G\left[V_{\frac{1}{2}}\right], w\right)=\frac{1}{2} w\left(V_{\frac{1}{2}}\right)$,

(ii) every minimum vertex cover $C$ for $(G, w)$ satisfies $V_{1} \subseteq C \subseteq V_{1} \cup V_{\frac{1}{2}}$ and $C \cap V_{\frac{1}{2}}$ is a minimum vertex cover for $\left(G\left[V_{\frac{1}{2}}\right], w\right)$,

(iii) every (minimum) vertex cover $C_{\frac{1}{2}}$ for $\left(G\left[V_{\frac{1}{2}}\right]\right.$,w) together with $V_{1}$ forms a (minimum) vertex cover for $(G, w)$,

(iv) $V_{0}=\cap_{y \in V C^{*}(G, w)} V_{0}^{y}, \quad V_{1}=\cap_{y \in V C^{*}(G, w)} V_{1}^{y}=\Gamma\left(V_{0}\right), V_{\frac{1}{2}}=V \backslash\left(V_{1} \cup V_{2}\right)$,

(v) $\emptyset \neq U \subseteq V_{1}$ implies $w\left(V_{0} \cap \Gamma(U)\right)>w(U)$.

In unweighted case its time complexity is $O(|E| \sqrt{|V|})$. Moreover, if $G$ is bipartite, then $V_{0}$ is the set of all vertices that are avoided by each minimum vertex cover for $(G, w), V_{1}$ is the intersection of all minimum vertex covers for $(G, w)$, and $v c\left(G\left[V_{\frac{1}{2}}\right], w\right)=\frac{1}{2} w\left(V_{\frac{1}{2}}\right)$. In particular, if $G$ is an unweighted bipartite graph, then $V_{0}{ }^{2}$ is the set of vertices in $G$ which are avoided by at least one maximum matching in $G$, and $G\left[V_{\frac{1}{2}}\right]$ has a perfect matching.

Lemma 1 shows in particular, that $v c^{*}(G, w)<\frac{1}{2} w(V)$ iff $V_{0}^{*}(G, w) \neq \emptyset$ iff there is an independent set $I$ in $G$ such that $w(\Gamma(I))<w(I)$; and any of these conditions implies $V_{0}(G, w) \neq \emptyset$. For us it is interesting to have the structural characterization of instances $(G, w)$ with $v c^{*}(G, w)=\frac{1}{2} w(V)$, as Min-w-VC reduces to such instances. Namely, we have that $v c^{*}(G, w)=\frac{1}{2} w(V)$ iff for every independent set $I$ in $G w(\Gamma(I)) \geq w(I)$ holds.

In the next section we refine the NT-Theorem in another direction and the Min- $w$-VC problem will be reduced to even more restricted instances $(G, w)$, namely those with $w>0$ for which $x \equiv \frac{1}{2}$ on $V$ is the unique element of $V C^{*}(G, w)$. They can be characterized similarly, using Lemma 1 , as those instances $(G, w)$ for which $w(\Gamma(I))>w(I)$ holds for every nonempty independent set $I$ in $G$.

\section{Decomposition into Irreducible Subgraphs}

The partition $V_{0}, V_{\frac{1}{2}}$, and $V_{1}$ of the vertex set $V$ from Theorem 2 satisfies $V_{1} \subseteq$ $C \subseteq V_{1} \cup V_{\frac{1}{2}}$ for every minimum vertex cover $C$. In this case the problem kernel $V_{\frac{1}{2}}$ is the largest among all $V_{\frac{1}{2}}^{y}, y \in V C^{*}(G, w)$, in fact $V_{\frac{1}{2}}=\cup_{y \in V C^{*}(G, w)} V_{\frac{1}{2}}^{y}$. On the other hand, in the original NT-Theorem it is natural to search for such decomposition with $V_{\frac{1}{2}}$ as small as possible. This is motivated by the fact that the Min-w-VC problem for $(G, w)$ reduces to the one for $\left(G\left[V_{\frac{1}{2}}\right], w\right)$. In what follows we will see that one can find in polynomial time $x \in V C^{*}(G, w)$ for which $V_{\frac{1}{2}}^{x}$ is the smallest among all $V_{\frac{1}{2}}^{y}, y \in V C^{*}(G, w)$, namely $V_{\frac{1}{2}}^{x}=\cap_{y \in V C^{*}(G, w)} V_{\frac{1}{2}}^{y}$. Furthermore, for the problem kernel $\left(G\left[V_{\frac{1}{2}}^{x}\right], w\right), z \equiv \frac{1}{2}$ on $V_{\frac{1}{2}}^{x}$ is the unique element of $V C^{*}\left(G\left[V_{\frac{1}{2}}^{x}\right], w\right)$. Also we assume from now on that $w>0$, as vertices with weight 0 are not contained in $\cap_{y \in V C^{*}(G, w)} V_{\frac{1}{2}}^{y}$.

The following theorem summarizes the main results of this section. 
Theorem 3. There exists a polynomial time algorithm (running in time $O(|E| \sqrt{|V|})$ in unweighted case) that for a graph $G=(V, E)$ with vertex weights $w: V \rightarrow(0, \infty)$ and $v c^{*}(G, w)=\frac{1}{2} w(V)$ constructs a partition

$$
V=K \bigcup \cup_{i=1}^{s} T_{i} \bigcup \cup_{i=1}^{s} S_{i}
$$

with the following properties:

(i) there is a minimum half-integral vertex cover $x$ for $(G, w)$ such that $V_{0}^{x}=$ $\cup_{i=1}^{s} T_{i}, V_{1}^{x}=\cup_{i=1}^{s} S_{i}$, and $V_{\frac{1}{2}}^{x}=K$,

(ii) $K=\cap_{y \in V C^{*}(G, w)} V_{\frac{1}{2}}^{y}$. Moreover, if $K \neq \emptyset, z \equiv \frac{1}{2}$ on $K$ is the unique element of $V C^{*}(G[K], w)$ and $v c(G[K], w)>v c^{*}(G[K], w)=\frac{1}{2} w(K)$.

(iii) For each $i \in\{1,2, \ldots, s\}$ the following holds true:

(a) $S_{i}=\Gamma\left(T_{i}\right) \backslash \cup_{j=1}^{i-1} S_{j}$,

(b) $w\left(T_{i}\right)=w\left(S_{i}\right)=v c\left(G\left[T_{i} \cup S_{i}\right], w\right)$,

(c) $\emptyset \neq T \subsetneq T_{i}$ implies $w\left(\Gamma(T) \cap S_{i}\right)>w(T)$,

(d) for every $C \in \operatorname{VC}(G, w) C \cap\left(T_{i} \cup S_{i}\right)$ is either $T_{i}$ or $S_{i}$, and if $S_{i}$ is not an independent set then $C \cap\left(T_{i} \cup S_{i}\right)=S_{i}$.

Remark 3. Under the assumptions of Theorem 3, we have $V_{0}^{*}(G, w)=V_{1}^{*}(G, w)=$ $\emptyset$ and we cannot say much, in general, about $V_{0}(G, w)$ and $V_{1}(G, w)$. But in many cases the theorem gives us nontrivial information about $V_{0}(G, w)$ and $V_{1}(G, w)$. Let the corresponding partition $V=K \bigcup \cup_{i=1}^{s} T_{i} \bigcup \cup_{i=1}^{s} S_{i}$ be fixed. We will say that $i \in\{1,2, \ldots, s\}$ is determined if for every $C \in V C(G, w), C \cap\left(T_{i} \cup S_{i}\right)=S_{i}$ (i.e., $S_{i} \subseteq C$ and $T_{i} \cap C=\emptyset$ ). By part (iii)(d) of Theorem 3 we know that any $i$ for which $S_{i}$ is not an independent set is determined. Also, if $i$ is such that for some $k>i$ there exists an edge between $S_{i}$ and $S_{k}$ as well as an edge between $S_{i}$ and $T_{k}$, then $i$ is determined. Further, if $i$ is determined and $j<i$ is such that there exists an edge between $T_{i}$ and $S_{j}$, then $j$ is determined as well. These observations allow in some cases to further reduce the kernel $\left(G\left[V_{\frac{1}{2}}\right], w\right)$ obtained in Theorem 2, as we can tell à priori for some $i$ that $T_{i} \subseteq V_{0}(G, w)$ and $S_{i} \subseteq V_{1}(G, w)$.

To explain some ideas behind the proof of Theorem 3, we study the Min$w$-VC problem on weighted bipartite graphs with bipartition $V=L \cup R$, and satisfying $v c(G, w)=\frac{1}{2} w(V)$. In this setting an edge $\{u, v\} \in E$ is called allowed for $(G, w)$ if for every minimum vertex cover $C$ for $(G, w)$ only one of the vertices $u$ and $v$ belongs to $C$, otherwise it is called forbidden. Further, $(G, w)$ is called elementary if it has exactly two minimum vertex covers, namely $L$ and $R$. Clearly, if $(G, w)$ is elementary then $G$ is connected and every edge of $G$ is allowed. The notions of an allowed edge and an elementary graph come from decomposition theorems related to maximum matchings in unweighted graphs. See [12, Thm. 4.1.1] for the proof that our notions are equivalent in case of unweighted bipartite graphs with perfect matching.

We need also to prove the following generalization to weighted graphs of the classical Dulmage-Mendelsohn Decomposition Theorem for bipartite graphs, where we are focused on minimum vertex covers rather than on maximum matchings. 
Theorem 4. Let $G=(V, E)$ be a bipartite graph with bipartition $V=L \cup R$ and vertex weight $w: V \rightarrow(0, \infty)$. Assume that $v c(G, w)=\frac{1}{2} w(V)$. The subgraph of $G$ consisting of all allowed edges for $(G, w)$ has components, called blocks, $B_{i}=G\left[L_{i} \cup R_{i}\right]$ for $i=1,2, \ldots, r$ (here $\cup_{i=1}^{r} L_{i}=L$ and $\cup_{i=1}^{r} R_{i}=R$ are partitions). Each weighted block $\left(B_{i}, w\right)$ is an elementary graph, and the ordering $B_{1}, B_{2}, \ldots, B_{r}$ can be chosen with the following property: every edge in $G$ between two blocks $B_{i}$ and $B_{j}$ with $i<j$ must have its $R$-vertex in $B_{i}$ and $L$ vertex in $B_{j}$. The decomposition into blocks and their admissible ordering can be constructed in polynomial time; in unweighted case in time $O(|E| \sqrt{|V|})$.

Remark 4. In Theorem $4 L$ and $R$ are always in $V C(G, w)$, but if $(G, w)$ is not elementary there are also "intermediate" minimum vertex covers. Namely, each $C_{i}:=\bigcup_{k=1}^{i} R_{k} \cup \bigcup_{k=i+1}^{r} L_{k}, \quad i=0,1, \ldots, r$, belongs to $V C(G, w)$.

Let us mention some of the consequences of Theorem 3. For a graph $G=$ $(V, E)$ with vertex weights $w: V \rightarrow\langle 0, \infty)$ we firstly apply Theorem 2 to obtain $\left(G\left[V_{\frac{1}{2}}\right], w\right)$ whose positive weighted vertices satisfy the assumption of Theorem 3. This reduces the Min- $w$-VC problem for $(G, w)$ to the one for $(G[K], w)$, for which $x \equiv \frac{1}{2}$ on $K$ is the unique element of $V C^{*}(G[K], w)$. Moreover, the difference $v c(G, w)-v c^{*}(G, w)$ is preserved. It is the same as $v c(G[K], w)-v c^{*}(G[K], w)$, which is zero iff $K=\emptyset$. Hence we have the following

Corollary 1. There is a polynomial time algorithm (of time complexity $O(|E| \sqrt{|V|})$ in unweighted case) that for a graph $G=(V, E)$ with vertex weights $w: V \rightarrow\langle 0, \infty)$ decides whether $v c(G, w)=v c^{*}(G, w)$, and if the equality holds, finds one minimum vertex cover for $(G, w)$.

Remark 5. Since a (maximum) independent set for $(G, w)$ is a complement of a (minimum) vertex cover for $(G, w)$, all results above can be translated in obvious way to the ones for the Maximum Weighted Independent Set problem.

To describe some of possible applications, we will confine ourselves to the unweighted version of the Minimum VerTex COVER problem in the rest of the paper.

\section{Parametrized Complexity and Vertex Covers}

The Minimum Vertex Cover problem and its variants play a very special role among fixed-parameter tractable problems. Let us recall the basic parametrized version of the problem:

Instance: A graph $G=(V, E)$ and a nonnegative integer $k$

Question (for decision version): Is there a vertex cover for $G$ with at most $k$ vertices?

Task (for search version): Either find a vertex cover for $G$ with at most $k$ vertices or report that no such vertex cover exists. 
Recently, there have been increasing interest and progress in lowering the exponential running time of algorithms that solve NP-hard optimization problems, like Min-VC, precisely. One of the most important methods employed in the development of efficient parametrized algorithms for such problems is reduction to a problem kernel. For the parametrized decision version of the vertex cover problem it means to apply an efficient preprocessing on the instance $(G, k)$ to construct another instance $\left(G_{1}, k_{1}\right)$, where $G_{1}$ is a subgraph of $G$ (the kernel), $k_{1} \leq k$, and $G_{1}$ has a vertex cover with at most $k_{1}$ vertices iff $G$ has a vertex cover with at most $k$ vertices. As observed in [5], the Nemhauser-Trotter Theorem allows to find efficiently a linear size problem kernel for Min-VC. Namely, there is an algorithm of running time $O\left(k|V|+k^{3}\right)$ that, given an instance $(G=(V, E), k)$, constructs another instance $\left(G^{\prime}=\left(V^{\prime}, E^{\prime}\right), k^{\prime}\right)$ with the following properties: $G^{\prime}$ is an induced subgraph of $G,\left|V^{\prime}\right| \leq 2 k^{\prime}, k^{\prime} \leq k$, and $G$ admits a vertex cover of size $k$ iff $G^{\prime}$ admits a vertex cover of size $k^{\prime}$. Clearly, using the same technique one can solve the parametrized search version of the vertex cover problem, or the problem: to find a minimum vertex cover of $G$ if $v c(G) \leq k$, or report that $v c(G)>k$.

Unlike the Nemhauser-Trotter Theorem, Theorem 2 can be used as efficient reduction to linear size problem kernel for the following problem: to find all minimum vertex covers if $v c(G) \leq k$ or report that $v c(G)>k$.

PARAMETRIZED All-Min-VC PROBlem

Instance: $(G=(V, E), k)$ and a nonnegative integer $k$

Task: Either find all minimum vertex covers for $G$ if $v c(G) \leq k$, or report that $v c(G)>k$.

Theorem 5. There is an algorithm of running time $O\left(k|V|+k^{3}\right)$ that for a given instance $(G=(V, E), k)$ either reports that $v c(G)>k$, or finds a partition $V=N \cup Y \cup V^{\prime}$ such that $G^{\prime}:=G\left[V^{\prime}\right], k^{\prime}:=k-|Y|, v c\left(G^{\prime}\right) \geq \frac{1}{2}\left|V^{\prime}\right|$, and $\left|V^{\prime}\right| \leq 2 k^{\prime}$. Moreover, $v c(G) \leq k$ iff $v c\left(G^{\prime}\right) \leq k^{\prime}$, and assuming $v c(G) \leq k$ :

(i) for every minimum vertex cover $C^{\prime}$ for $G^{\prime}: C^{\prime} \cup Y \in V C(G)$, and

(ii) for every minimum vertex cover $C$ for $G: Y \subseteq C \subseteq Y \cup V^{\prime}$ and $C \cap V^{\prime} \in$ $V C\left(G^{\prime}\right)$.

Proof. Let an instance $(G=(V, E), k)$ be given. Clearly, every vertex $v \in V$ of degree at least $k+1$ has to belong to every vertex cover of size at most $k$, provided $v c(G) \leq k$. Denote $Y^{\prime \prime}$, the set of vertices of $G$ of degree at least $(k+1), N^{\prime \prime}$, the set of isolated vertices of $G \backslash Y^{\prime \prime}, V^{\prime \prime}:=V \backslash\left(Y^{\prime \prime} \cup N^{\prime \prime}\right)$, and $k^{\prime \prime}=k-\left|Y^{\prime \prime}\right|$. Firstly, in running time $O(k|V|)$ we can construct a graph $G^{\prime \prime}=\left(V^{\prime \prime}, E^{\prime \prime}\right):=G\left[V^{\prime \prime}\right]$ (see, e.g., Buss [2] for such simple algorithm). Clearly, $v c(G) \leq k$ iff $v c\left(G^{\prime \prime}\right) \leq k^{\prime \prime}$, and assuming $v c(G) \leq k$ : (i) for every $C^{\prime \prime} \in V C\left(G^{\prime \prime}\right), C^{\prime \prime} \cup Y^{\prime \prime} \in V C(G)$, and (ii) for every $C \in V C(G), Y^{\prime \prime} \subseteq C \subseteq Y^{\prime \prime} \cup V^{\prime \prime}$ and $C \cap V^{\prime \prime} \in V C\left(G^{\prime \prime}\right)$.

Each vertex of $G^{\prime \prime}$ has degree at most $k$. Hence $v c\left(G^{\prime \prime}\right) \leq k^{\prime \prime}$ is only possible if $\left|E^{\prime \prime}\right| \leq k k^{\prime \prime}$. If $\left|E^{\prime \prime}\right|>k k^{\prime \prime}$, we can report that $v c(G)>k$ and the algorithm terminates. Otherwise, we have $\left|E^{\prime \prime}\right| \leq k \cdot k^{\prime \prime}\left(\leq k^{2}\right)$, and since $G^{\prime \prime}$ does not contain isolated vertices, it follows that $\left|V^{\prime \prime}\right| \leq 2\left|E^{\prime \prime}\right| \leq 2 k^{2}$. Now we apply Theorem 2 to the graph $G^{\prime \prime}$ (with $w \equiv 1$ ). Namely, we partition the vertex set 
$V^{\prime \prime}$ into three subsets $V_{0}, V_{1}, V_{\frac{1}{2}}$ in time $O\left(\left|E^{\prime \prime}\right| \sqrt{\left|V^{\prime \prime}\right|}\right)=O\left(k^{3}\right)$. Further, we put $Y:=Y^{\prime \prime} \cup V_{1}, N:=N^{\prime \prime} \cup V_{0}, V^{\prime}:=V_{\frac{1}{2}}, G^{\prime}:=G\left[V^{\prime}\right]$, and $k^{\prime}:=k^{\prime \prime}-\left|V_{1}\right|=k-|Y|$. Obviously, $v c(G) \leq k$ iff $v c\left(G^{\prime}\right) \leq k^{\prime}$, and from Theorem 2 also $v c\left(G^{\prime}\right) \geq \frac{1}{2}\left|V^{\prime}\right|$. It means if $\left|V^{\prime}\right|>2 k^{\prime}$, we can report that $v c\left(G^{\prime}\right)>k^{\prime}$, hence $v c(G)>k$, and the algorithm terminates. Otherwise $\left|V^{\prime}\right| \leq 2 k^{\prime}$ holds, as was required. All other properties follow directly from Theorem 2 .

Theorem 5 can be used to many other parametrized problems related to Min-VC as reduction to linear size problem kernel. The typical example is the problem, whose task is to find one minimum vertex cover for $G$ under some additional constraints.

Parametrized Constrained-Min-VC Problem

Instance: $(G=(V, E), k), k$ a nonnegative integer, and finitely many linear constraints $P_{1}, P_{2}, \ldots, P_{r}$ of the form $P_{i}: \sum_{v \in V} a_{i}(v) x(v) \leq b_{i}, i=1,2, \ldots, r$, where $a_{i}(v), b_{i} \in \mathbb{R}$.

Task: If $v c(G) \leq k$ find $C$ from $V C(G)$, whose indicator function $x=x^{C}$ satisfies all constraints $P_{1}, P_{2}, \ldots, P_{r}$, otherwise report that no such minimum vertex cover exists.

The most natural case is when each $a_{i}(v)$ is either 0 or 1 , and $b_{i}$ are nonnegative integers. Then constraint $P_{i}$ says, that $\left|C \cap A_{i}\right| \leq b_{i}$ for a set $A_{i}:=\{v \in V$ : $\left.a_{i}(v)=1\right\}$ and for a vertex cover $C \in V C(G)$ to be found. The problem has received considerable attention even in its very simplified version, when $G=(V, E)$ is a bipartite graph with bipartition $(L, R)$, and two nonnegative integers $k_{\mathrm{L}}$ and $k_{\mathrm{R}}$ (with $k=k_{\mathrm{L}}+k_{\mathrm{R}}$ ) are given as an input. The $k_{\mathrm{L}}$ and $k_{\mathrm{R}}$ represent constraints $|C \cap L| \leq k_{\mathrm{L}},|C \cap R| \leq k_{\mathrm{R}}$ on $C \in V C(G)$ to be found. This problem arises from the extensively studied fault coverage problem for reconfigurable memory arrays in VLSI design, see [5] and references therein.

Theorem 5 clearly allows efficient reduction to the linear size problem kernel for Parametrized Constrained Min-VC. Namely, $(G=(V, E), k)$ with constraints $P_{i}: \sum_{v \in V} a_{i}(v) x(v) \leq b_{i}, i=1,2, \ldots, r$ is reduced using Theorem 5 to $\left(G^{\prime}=\left(V^{\prime}, E^{\prime}\right), k^{\prime}\right)$ with $\left|V^{\prime}\right| \leq 2 k^{\prime}(\leq 2 k)$, and with constraints $P_{i}^{\prime}: \sum_{v \in V^{\prime}} a_{i}(v) x(v) \leq b_{i}^{\prime}\left(:=b_{i}-\sum_{v \in Y} a_{i}(v)\right), i=1,2, \ldots, r$.

\section{Further research}

Seemingly a new technique, called the crown reduction (also crown decomposition, crown rules) has been recently introduced in [6] and [1] for the (unweighted) VerTex Cover problem. A crown decomposition in a graph $G=(V, E)$ is a partitioning of the vertex set $V$ into three sets $V_{0}, V_{1}$, and $V_{\frac{1}{2}}$ satisfying the following conditions: (1) $V_{0}$ (the crown) is an independent set; (2) $V_{1}$ (the head) separates $V_{0}$ from $V_{\frac{1}{2}}$ (the rest), i.e., there are no edges between $V_{0}$ and $V_{\frac{1}{2}}$; and (3) there is a matching of $\left|V_{1}\right|$ edges from $V_{1}$ to $V_{0}$.

It can be seen from Lemma 1 that any minimum half-integral vertex cover $x$ defines such partition (as (3) easily follows from Lemma 1(iv)). Hence we not only generalize NT Theorem, but also link this well studied theorem very close to the 
crown reduction technique. From this link and our theorems it also easily follows that if a graph admits a crown decomposition, then a crown decomposition can be computed in polynomial time. Applying reduction rules of Theorem 2 and Theorem 3 one can obtain an irreducible instance in which $|\Gamma(I)|>|I|$ for every nonempty independent set $I$.

Let us mention that the crown reduction technique can be applied effectively to other parametrized problems (see [3]). Moreover, our new decomposition theorems for vertex covers have connections with "parametrized enumeration" in the sense of listing all minimal solutions, as also discussed in [8]. We believe that the technique of this paper may be a powerful tool to designing algorithms for other fixed parameter tractable problems that are related to the Min-VC problem.

Acknowledgments. We are thankful to an anonymous referee for useful comments.

\section{References}

1. F. N. Abu-Khzam, R. L. Collins, M. R. Fellows, M. A. Langston, W. H. Suters and C. T. Symons, Kernelization Algorithms for the Vertex Cover Problem: Theory and Experiments, Proceeding of Workshop on Algorithm Engineering and Experiments (ALENEX), New Orleans, Louisiana, January, 2004.

2. J. F. Buss and J. Goldsmith, Nondeterminism within P, SIAM Journal on Computing 22(3)(1993), 560-573.

3. B. Chor, M. Fellows and D. Juedes, An efficient FPT algorithm for saving $k$ colors, Manuscript, 2003.

4. J. Chen, I. A. Kanj and W. Jia, Vertex cover: further observations and further improvements, Journal of Algorithms 41(2001), 280-301.

5. J. Chen and I. A. Kanj, On constrained minimum vertex covers of bipartite graphs: improved algorithms, Journal of Computer Systems Sci. 67(2003), 833-847.

6. F. Dehne, M. Fellows and F. Rosamond, An FPT algorithm for set splitting, LNCS 2880 (WG'03), pp. 180-191, 2003.

7. I. Dinur and S. Safra, The importance of being biased, STOC, 2002, pp. 33-42.

8. H. Fernau, On parameterized enumeration, LNCS 2383 (COCOON'02), pp. 564$573,2002$.

9. A. V. Goldberg and R. E. Tarjan, A new approach to the maximum-flow problem, Journal of ACM 35(1988), 921-940.

10. J. Hopcroft and R. M. Karp, An $O\left(n^{2.5}\right)$ algorithm for maximum matching in bipartite graphs, SIAM J. Computing 2(1973), 225-231.

11. E. L. Lawler, Combinatorial Optimization: Networks and Matroids, Holt, Rinehart, and Winston, 1976.

12. L. Lovász and M. D. Plummer, Matching Theory, North-Holland, Amsterdam, 1986.

13. S. Micali and V. V. Vazirani, An $O(\sqrt{|V|}|E|)$ algorithm for finding maximum matching in general graphs, Proc. of 21st IEEE Symposium on Foundation of Computer Science, Syracuse, New York, 1980, pp. 17-27.

14. G. L. Nemhauser and L. E. Trotter, Vertex packings: structural properties and algorithms, Math. Programming 8(1975), 232-248.

15. R. Niedermeier and P. Rossmanith, On efficient fixed parameter algorithms for weighted vertex cover, Journal of Algorithms 47(2)(2003), 63-77. 